PROCEEDINGS OF THE

AMERICAN MATHEMATICAL SOCIETY

Volume 135, Number 6, June 2007, Pages 1769-1773

S 0002-9939(06)08666-7

Article electronically published on November 7, 2006

\title{
EXTENSION OF POLYNOMIALS AND JOHN'S THEOREM FOR SYMMETRIC TENSOR PRODUCTS
}

\author{
DANIEL CARANDO AND VERÓNICA DIMANT
}

(Communicated by N. Tomczak-Jaegermann)

\begin{abstract}
We show that for every infinite-dimensional normed space $E$ and every $k \geq 3$ there are extendible $k$-homogeneous polynomials which are not integral. As a consequence, we prove a symmetric version of a result of John.
\end{abstract}

\section{INTRODUCTION}

Every continuous linear functional defined over a normed space $E$ can be extended to any superspace $F \supset E$, via the Hahn-Banach Theorem. For continuous $k$-homogeneous polynomials or $k$-linear forms (with $k \geq 2$ ) such an extension is not always possible. This is related to the fact that the projective tensor norm $\pi_{s}$ is not injective. Extendible polynomials are defined in [15] as those admitting an extension to any larger space. They can be seen as the dual of the $k$-fold symmetric tensor product of $E$ with an injective tensor norm $\eta_{s}$ [7, 3, 15] given by

$$
\bigotimes_{s, \eta_{s}}^{k} E \stackrel{1}{\hookrightarrow} \bigotimes_{s, \pi_{s}}^{k} \ell_{\infty}\left(B_{E^{\prime}}\right) \text {. }
$$

Since the symmetric tensor norm $\varepsilon_{s}$ is injective, every integral polynomial is extendible. Thus, it is natural to ask about the existence of extendible nonintegral polynomials. In this direction, 4] and 6] proved independently that every 2-homogeneous extendible polynomial over a cotype 2 space is integral. On the other hand, there exist extendible nonintegral $k$-homogeneous polynomials on $\ell_{p}$, for every $p>2$ and $k \geq 2$ (see, for example, [5]). These two facts focus the question on the existence of an infinite-dimensional space where all the extendible $k$-homogeneous polynomials are integral, for some $k \geq 3$. Recently, Pérez-García [16] proved that if $k \geq 4$ there is no such space. We fill in the gap by proving the same result for $k \geq 3$.

In [12, Grothendieck asked about the existence of nonnuclear locally convex spaces $E$ and $F$ such that the injective and projective norm are equivalent on $E \otimes F$ and conjectured a negative answer. Pisier [17] provided a counterexample exhibiting a Banach space $P$ such that $P \otimes_{\varepsilon} P \simeq P \otimes_{\pi} P$. In [13, 14, John proved

Received by the editors August 16, 2005 and, in revised form, February 1, 2006.

2000 Mathematics Subject Classification. Primary 46G25, 46 B28.

Key words and phrases. Extendible polynomials, symmetric tensor products, Grothendieck's conjecture.

The first author was partially supported by CONICET Resolución No. 1584-04, UBACyT Grant X058 and ANPCyT PICT 03-15033.

(C)2006 American Mathematical Society 1769 
that such an example is not possible for tensor products of order $k \geq 3$. For locally convex spaces he showed that $\bigotimes_{\varepsilon}^{k} E \simeq \bigotimes_{\pi}^{k} E$ if and only if $E$ is nuclear. For normed spaces, the conclusion also holds for tensor products of different spaces.

Theorem 1.1 ([13]). If the norms $\varepsilon$ and $\pi$ are equivalent in the tensor product $\bigotimes_{i=1}^{k} E_{i}$, then all but two of the spaces $E_{1}, \ldots, E_{k}$ are finite dimensional.

The fact that extendible and integral $k$-homogeneous polynomials never coincide for $k \geq 3$ means, in the tensor setting, that the norms $\eta_{s}$ and $\varepsilon_{s}$ are never equivalent for $k$-fold symmetric tensor products. Therefore, the norms $\pi_{s}$ and $\varepsilon_{s}$ are not equivalent either, and thus we obtain the symmetric version of John's result for normed spaces.

We refer to [7, 9] and [10] for notation and terminology on tensor products, polynomials and multilinear mappings on normed spaces.

\section{The RESUlts}

In [2, Boas showed estimates for the Bohr radius on $\ell_{p}^{n}:=\left(\mathbb{C}^{n},\|\cdot\|_{p}\right)$. For the upper bounds he proved, for each $p$, the existence of a symmetric $k$-linear form on $\ell_{p}^{n}$ as in (2.1) below with "small" norm. For our purpose, we need a symmetric $k$ linear form having small norm on $\ell_{2}^{n}$ and $\ell_{\infty}^{n}$ simultaneously. Therefore, we slightly change the proof of Boas to obtain the following lemma.

Lemma 2.1. For each $n \geq 2$ there exists a symmetric $k$-linear form $T_{n} \in \mathcal{L}\left({ }^{k} \mathbb{C}^{n}\right)$ of the form

$$
T_{n}\left(x_{1}, \ldots, x_{k}\right)=\sum_{j_{1}, \ldots, j_{k}=1}^{n} \varepsilon_{j} \cdot x_{1}\left(j_{1}\right) \cdots x_{k}\left(j_{k}\right),
$$

where $\varepsilon_{j}= \pm 1, j=\left(j_{1}, \ldots, j_{k}\right)$ and $\varepsilon_{j}=\varepsilon_{\sigma(j)}$ for all permutations $\sigma$ that verifies

$$
\begin{aligned}
\left\|T_{n}\right\|_{\mathcal{L}\left({ }^{k} \ell_{2}^{n}\right)} & \leq \sqrt{32 k \log (6 k) k !} n^{1 / 2}, \\
\left\|T_{n}\right\|_{\mathcal{L}\left({ }^{k} \ell_{\infty}^{n}\right)} & \leq 2 \sqrt{32 k \log (6 k) k !} n^{k / 2+1 / 2} .
\end{aligned}
$$

Proof. To each choice of $j_{1} \leq j_{2} \leq \cdots \leq j_{k}$ we assign a different Rademacher function $r_{j}$. We consider for each $t \in[0,1]$ the $k$-linear form on $\mathbb{C}^{n} \times \cdots \times \mathbb{C}^{n}$ given by

$$
F_{t}\left(x_{1}, \ldots, x_{k}\right)=\sum_{j_{1} \leq j_{2} \leq \cdots \leq j_{k}} r_{j}(t) \sum_{\sigma} x_{1}\left(\sigma\left(j_{1}\right)\right) \cdots x_{k}\left(\sigma\left(j_{k}\right)\right) .
$$

In the proof of [2, Theorem 4], it is shown that for every positive constant $R_{2}, \lambda_{2}$, $R_{\infty}, \lambda_{\infty}$ the following inequalities hold:

$$
\begin{gathered}
\mu\left\{t \in[0,1]:\left\|F_{t}\right\|_{\left.\mathcal{L}_{\left(k \ell_{2}^{n}\right)}>2 \sqrt{2} R_{2}\right\}} \leq 4(1+4 k)^{2 n k} e^{-R_{2} \lambda_{2}+\frac{1}{2} \lambda_{2}^{2} k !},\right. \\
\mu\left\{t \in[0,1]:\left\|F_{t}\right\|_{\mathcal{L}\left({ }^{k} \ell_{\infty}^{n}\right)}>2 \sqrt{2} R_{\infty}\right\} \leq 4(1+4 k)^{2 n k} e^{-R_{\infty} \lambda_{\infty}+\frac{1}{2} \lambda_{\infty}^{2} k ! n^{k}},
\end{gathered}
$$

where $\mu$ denotes the Lebesgue measure on $[0,1]$.

Choosing, as in [2], $R_{2}=\sqrt{2 k ! \log \left(8(1+4 k)^{2 n k}\right)}$ and $\lambda_{2}=\frac{R_{2}}{k !}$, one obtains

$$
\mu\left\{t \in[0,1]:\left\|F_{t}\right\|_{\mathcal{L}\left(k \ell_{2}^{n}\right)}>2 \sqrt{2} R_{2}\right\} \leq \frac{1}{2} .
$$


Also, for $R_{\infty}=\sqrt{2 k ! n^{k} \log \left(8(1+4 k)^{2 n k}\right)}$ and $\lambda_{\infty}=\frac{R_{\infty}}{k ! n^{k}}$, we have

$$
\mu\left\{t \in[0,1]:\left\|F_{t}\right\|_{\mathcal{L}\left({ }^{k} \ell_{\infty}^{n}\right)}>2 \sqrt{2} R_{\infty}\right\} \leq \frac{1}{2} .
$$

Since the right-hand side of inequality (2.5) is a decreasing function of $R_{\infty}$ (for a fixed $\lambda_{\infty}$ ), if we take $\widetilde{R}_{\infty}=2 R_{\infty}$, we obtain

$$
\mu\left\{t \in[0,1]:\left\|F_{t}\right\|_{\left.\mathcal{L}^{k} \ell_{\infty}^{n}\right)}>2 \sqrt{2} \widetilde{R}_{\infty}\right\}<\frac{1}{2} .
$$

Therefore, $\left\|F_{t}\right\|_{\mathcal{L}\left({ }^{k} \ell_{2}^{n}\right)} \leq 2 \sqrt{2} R_{2}$ and $\left\|F_{t}\right\|_{\left.\mathcal{L}^{(k} \ell_{\infty}^{n}\right)} \leq 2 \sqrt{2} \widetilde{R}_{\infty}$ simultaneously for $t$ in a positive measure set. For any such $t$ we can define $T_{n}=F_{t}$, which verifies inequalities (2.2) and (2.3) since $8(1+4 k)^{2 n k}<(6 k)^{2 n k}$, for all $n, k \geq 2$.

Since $\ell_{\infty}^{n}$ is 1-complemented in any larger space, the usual and the extendible norms coincide in $\mathcal{P}\left({ }^{k} \ell_{\infty}^{n}\right)$. This enables us to derive from the previous lemma the following result (where $\mathcal{P}_{e}$ denotes the space of extendible polynomials and $\mathcal{P}_{I}$ the space of integral polynomials).

Lemma 2.2. For each $n \in \mathbb{N}$ there exists a $k$-homogeneous polynomial $P_{n} \in \mathcal{P}\left({ }^{k} \ell_{2}^{n}\right)$ such that

$$
\left\|P_{n}\right\|_{\mathcal{P}_{e}\left({ }^{k} \ell_{2}^{n}\right)} \leq C n^{k / 2+1 / 2} \quad \text { and } \quad\left\|P_{n}\right\|_{\mathcal{P}_{I}\left({ }^{k} \ell_{2}^{n}\right)} \geq D n^{k-1 / 2},
$$

where $C$ and $D$ are positive constants independent of $n$.

Proof. Let $P_{n}$ be the $k$-homogeneous polynomial associated to the symmetric $k$ linear form defined in the previous lemma. We have

$$
\begin{aligned}
\left\|P_{n}\right\|_{\mathcal{P}_{e}\left({ }^{k} \ell_{2}^{n}\right)} & \leq\left\|P_{n}\right\|_{\mathcal{P}_{e}\left({ }^{k} \ell_{\infty}^{n}\right)}\left\|i d: \ell_{2}^{n} \rightarrow \ell_{\infty}^{n}\right\|^{k}=\left\|P_{n}\right\|_{\mathcal{P}\left({ }^{k} \ell_{\infty}^{n}\right)} \\
& \leq\left\|T_{n}\right\|_{\mathcal{L}\left({ }^{k} \ell_{\infty}^{n}\right)} \leq C n^{k / 2+1 / 2} .
\end{aligned}
$$

For the other inequality, we define the symmetric tensor $s_{n} \in \bigotimes_{s}^{k} \ell_{2}^{n}$ by

$$
s_{n}=\sum_{j} \varepsilon_{j} e_{j_{1}} \otimes \cdots \otimes e_{j_{k}},
$$

where $\varepsilon_{j}$ are the same signs as in the definition of $T_{n}$. Since

$$
n^{k}=P_{n}\left(s_{n}\right) \leq\left\|P_{n}\right\|_{\mathcal{P}_{I}\left({ }^{k} \ell_{2}^{n}\right)}\left\|s_{n}\right\|_{\bigotimes_{s, \varepsilon_{s}}^{k} \ell_{2}^{n}}
$$

and

$$
\left\|s_{n}\right\|_{\otimes_{s, \varepsilon_{s}}^{k} \ell_{2}^{n}}=\left\|P_{n}\right\|_{\mathcal{P}\left({ }^{k} \ell_{2}^{n}\right)} \leq\left\|T_{n}\right\|_{\mathcal{L}\left({ }^{k} \ell_{2}^{n}\right)},
$$

from inequality (2.2) we obtain the desired result.

Now we use Dvoretzky's theorem to extrapolate the situation in $\ell_{2}^{n}$ to an arbitrary infinite-dimensional normed space.

Theorem 2.3. For any infinite-dimensional normed space $E$ and any $k \geq 3$, there are extendible $k$-homogeneous polynomials on $E$ which are not integral.

Proof. Suppose $\mathcal{P}_{e}\left({ }^{k} E\right)=\mathcal{P}_{I}\left({ }^{k} E\right)$. Then, there must be a constant $M>0$ such that $\|P\|_{\left.\mathcal{P}_{I}{ }^{k} E\right)} \leq M\|P\|_{\left.\mathcal{P}_{e}{ }^{k} E\right)}$ for all extendible polynomial on $E$. If $F \subset E$ is a subspace, any extendible polynomial on $F$ extends to an extendible polynomial on $E$ with the same extendible norm. Therefore, every extendible polynomial $Q$ on $F$ is integral and

$$
\|Q\|_{\mathcal{P}_{I}\left({ }^{k} F\right)} \leq M\|Q\|_{\mathcal{P}_{e}\left({ }^{k} F\right)} .
$$


By Dvoretzky's theorem, for each $n$ there exists an $n$-dimensional subspace $F_{n} \subset$ $E$ and an isomorphism $j_{n}: \ell_{2}^{n} \rightarrow F_{n}$ with $\left\|j_{n}\right\|=1$ and $\left\|j_{n}^{-1}\right\| \leq 2$. Let $P_{n} \in \mathcal{P}\left({ }^{k} \ell_{2}^{n}\right)$ be as in the previous lemma and define $Q_{n}=P_{n} \circ j_{n}^{-1} \in \mathcal{P}\left({ }^{k} F_{n}\right)$. We have

$$
\left\|Q_{n}\right\|_{\mathcal{P}_{e}\left({ }^{k} F_{n}\right)} \leq\left\|P_{n}\right\|_{\mathcal{P}_{e}\left({ }^{k} \ell_{2}^{n}\right)}\left\|j_{n}^{-1}\right\|^{k} \leq 2^{k} C n^{k / 2+1 / 2} .
$$

On the other hand,

$$
D n^{k-1 / 2} \leq\left\|P_{n}\right\|_{\mathcal{P}_{I}\left({ }^{k} \ell_{2}^{n}\right)} \leq\left\|Q_{n}\right\|_{\mathcal{P}_{I}\left({ }^{k} F_{n}\right)} .
$$

By (2.6), we obtain

$$
D n^{k-1 / 2} \leq M 2^{k} C n^{k / 2+1 / 2} \quad \text { for all } n \in \mathbb{N},
$$

which is impossible if $k \geq 3$.

Clearly, the above result is also valid for $k$-linear forms. Moreover, with slight modifications we can show the existence of nonintegral extendible $k$-linear forms on the product of different normed spaces, provided three of them are infinite dimensional. So we have:

Theorem 2.4. If every extendible $k$-linear form on $E_{1} \times \cdots \times E_{k}$ is integral, then all the spaces but two are finite dimensional.

As an application of the previous theorems, first we note that Theorem 1.1 can be deduced from Theorem 2.4. Actually, we have

Corollary 2.5. If the norms $\varepsilon$ and $\eta$ are equivalent in the tensor product $\bigotimes_{i=1}^{k} E_{i}$, then all but two of the spaces $E_{1}, \ldots, E_{k}$ are finite dimensional.

As for the symmetric version of this result, Floret [1] proved it for $E=L_{\infty}(\mu)$. It is also simple to prove it for stable Banach spaces (i.e., spaces $E$ such that $E \times E \simeq E$ ). Indeed, for these spaces, $\bigotimes_{s, \varepsilon_{s}}^{k} E \simeq \bigotimes_{\varepsilon}^{k} E$ and $\bigotimes_{s, \pi_{s}}^{k} E \simeq \bigotimes_{\pi}^{k} E$. Therefore, for stable spaces, the symmetric version follows from the result for the full tensor product. As a consequence of Theorem 2.3, we obtain this result for arbitrary normed spaces.

Corollary 2.6. For $k \geq 3$, the norms $\varepsilon_{s}$ and $\eta_{s}$ are equivalent in the symmetric tensor product $\bigotimes_{s}^{k} E$ if and only if $E$ is finite dimensional. In particular, $\bigotimes_{s, \varepsilon_{s}}^{k} E \simeq$ $\bigotimes_{s, \pi_{s}}^{k} E$ if and only if $E$ is finite dimensional.

\section{REFERENCES}

[1] Ansemil, José and Floret, Klaus. The symmetric tensor product of a direct sum of locally convex spaces, Studia Math. 129 (1998), 285-295. MR1609655 (99b:46003)

[2] Boas, Harold P. Majorant series, J. Korean Math. Soc. 37 (2000), no. 2, 321-337. MR.1775963 (2001j:32001)

[3] Carando, Daniel. Extendible polynomials on Banach spaces, J. Math. Anal. Appl. 233 (1999), no. 1, 359-372. MR1684392 (2000d:46057)

[4] - Extendibility of polynomials and analytic functions on $\ell_{p}$, Studia Math. 145 (2001), no. 1, 63-73. MR.1828993 (2002d:46042)

[5] Carando, Daniel; Dimant, Verónica and Sevilla-Peris, Pablo. Limit orders and multilinear forms on $\ell_{p}$ spaces, Publ. Res. Inst. Math. Sci., 42, no. 2, (2006), 507-522.

[6] Castillo, Jesús M. F.; García, Ricardo and Jaramillo, Jesús A. Extension of bilinear forms on Banach spaces, Proc. Amer. Math. Soc. 129 (2001), no. 12, 3647-3656. MR1860499 (2002i:46017)

[7] Defant, Andreas and Floret, Klaus. Tensor norms and operator ideals. North-Holland, Amsterdam, 1993. MR 1209438(94e:46130) 
[8] Díaz, Juan Carlos and Dineen, Seán. Polynomials on stable spaces, Ark. Mat. 36 (1998), 87-96. MR.1611149 (99a:46082)

[9] Dineen, Seán. Complex analysis on infinite-dimensional spaces. Springer Monographs in Mathematics. Springer-Verlag London, Ltd., London, 1999. MR.1705327 (2001a:46043)

[10] Floret, Klaus. Natural norms on symmetric tensor products of normed spaces, Note Mat. 17 (1997), 153-188. MR1749787 (2001g:46038)

[11] _ On ideals of $n$-homogeneous polynomials on Banach spaces, in Topological algebras with applications to differential geometry and mathematical physics (Athens, 1999), pages 19-38. Univ. Athens, Athens, 2002. MR2000732 (2004i:46075)

[12] Grothendieck, Alexandre. Résumé de la théorie métrique des produits tensoriels topologiques; Bol. Soc. Mat. São Paulo 8 (1956), 83-110. MR0094682 (20:1194)

[13] John, Kamil. Tensor product of several spaces and nuclearity, Math. Ann. 269 (1984), no. 3, 333-356. MR0761310 (86b:46119)

[14] _ Tensor powers of operators and nuclearity, Math. Nachr. 129 (1986), 115-121. MR0864627 (87m:46147)

[15] Kirwan, Padraig and Ryan, Ray. Extendibility of homogeneous polynomials on Banach spaces, Proc. Amer. Math. Soc. 126 (1992), 1023-1029. MR1415346 (98f:46042)

[16] Pérez-García, David. A counterexample using 4-linear forms, Bull. Aust. Math. Soc. 70 (2004), no. 3, 469-473. MR2103978 (2005g:46088)

[17] Pisier, Gilles. Counterexamples to a conjecture of Grothendieck, Acta Math. 151 (1983) 181208. MR0723009 (85m:46017)

Departamento de Matemática, Universidad de San Andrés, Vito Dumas 284, B1644BiD Victoria, Buenos Aires, Argentina

Current address: Departamento de Matemática - Pab I, Facultad de Cs. Exactas y Naturales, Universidad de Buenos Aires, (1428) Buenos Aires, Argentina

E-mail address: daniel@udesa.edu.ar, dcarando@dm.uba.ar

Departamento de Matemática, Universidad de San Andrés, Vito Dumas 284, B1644BiD Victoria, Buenos Aires, Argentina

E-mail address: vero@udesa.edu.ar 